\title{
Micro-abrasion resistance of thermochemically treated steels in aqueous solutions: Mechanisms, maps, materials selection
}

\author{
M.T. Mathew ${ }^{\mathrm{a}, *}$, M.M. Stack ${ }^{\mathrm{b}}$, B. Matijevic ${ }^{\mathrm{b}}$, L.A. Rocha ${ }^{\mathrm{a}, \mathrm{c}}$, E. Ariza ${ }^{\mathrm{a}}$ \\ ${ }^{a}$ Research Centre on Interfaces and Surfaces Performance, Azurém, 4800-058 Guimarães, Portugal \\ ${ }^{\mathrm{b}}$ Department Mechanical Engineering, University of Strathclyde, James Weir Building, 75 Montrose Street, Glasgow, G1 2 XJ, UK \\ ${ }^{\mathrm{c}}$ Department of Engineering, Universidade do Minho, Mecânica, Azurém, 4800-058 Guimarães, Portugal
}

Received 25 September 2006; received in revised form 18 June 2007; accepted 3 July 2007

Available online 5 September 2007

\begin{abstract}
The area of micro-abrasion is an interesting and relatively recent area in tribo-testing methodologies, where small particles of less than $10 \mu \mathrm{m}$ are employed between interacting surfaces. It is topical for a number of reasons; its direct relation to the mechanisms of the wear process in bio-tribological applications, ease in conducting tests and the good repeatability of the test results. It has widespread applications in conditions used in the space and offshore industries to bio-engineering for artificial joints and implants.

There have been many recent studies on the micro-abrasion performance of materials, ranging from work basic metals to nanostructured coatings. However, no significant work is reported on the micro-abrasion resistance of thermochemically treated steels. Hence, this paper looks at the performance of two thermochemically treated steels, Tenifer bath nitride stainless steel (T-SS) and vanadized carbon steel (V-CS) in such conditions with reference to the stainless steel (SS) by varying the applied load and sliding distance.

The results indicated that T-SS demonstrates exceptionally poor resistance to micro-abrasion. It was observed that the heat treatment process and properties of the hardened layer (hardness and thickness) are extremely important in determining the micro-abrasion resistance of such steels. Finally, the results were used to develop micro-abrasion mechanism and wastage maps, which can be used to optimize the surface treated materials for micro-abrasion resistance.
\end{abstract}

(C) 2007 Elsevier Ltd. All rights reserved.

Keywords: Thermochemical treatment; Tenifer; Vanadizing; Maps; Micro-abrasion; Mechanisms

\section{Introduction}

In sliding wear there are various test approaches to assess the motion of surfaces in contact with each other $[1,2]$. The study of micro-abrasion is a relatively recent technique developed to study the interaction of a third body in the contact, typically particles less than $10 \mu \mathrm{m}$ in size $[3,4]$. It has distinct advantages over many other kinds of abrasive wear tests namely the ease in conducting the experiment on a relatively small scale [5-7].

Many studies have been conducted on the microabrasion of materials ranging from basic metals to nano-structured films all of which have many practical

\footnotetext{
${ }^{*}$ Corresponding author.

E-mail address: mathew@dem.uminho.pt (M.T. Mathew).
}

applications; especially in bio-engineering [4-10]. Some efforts have also been made to employ a mapping approach in micro-abrasion, which can indicate the mechanisms of wear loss as a function of selected variables in the test [8-10]. Recently, work has been carried out to model the micro-abrasion process by considering the effects of the various parameters involved [11-13].

However, to date there has been no attempt to investigate the micro-abrasion performance of case hardened steels, despite their potential use in many practical applications, such as in power plants and in the automobile industry. Hence, this paper investigates the micro-abrasion performance of two case hardened thermochemically treated steels, namely Tenifer bath nitrided stainless steel (denoted as T-SS) and vanadized carbon steel (denoted as V-CS) in the presence of silicon carbide $(\mathrm{SiC})$ 


\section{Nomenclature}

SS untreated stainless steel

T-SS Tenifer bath nitrided stainless steel

$\mathrm{V}-\mathrm{CS}$

vanadized carbon steel.
$V \quad$ wear volume
$d \quad$ penetration depth
$b \quad$ crater diameter
$R \quad$ radius of the ball

slurry by varying the sliding distance and applied load. The tests accelerate the wear process and also simulate microabrasion in practical applications, such as in pumps and valves etc., where such heat treated steels can be employed to improve wear resistance. Mechanisms of micro-abrasion were identified based on the test conditions. Finally, microabrasion maps were constructed to demonstrate the change in mechanisms, wastage rate and the optimum material for the conditions of interest in the study.

\section{Experimental details}

\subsection{Thermochemical treatment of samples}

The samples of thermochemically treated steels were supplied by the Department of Materials Science of Faculty of Mechanical Engineering and Naval Architecture, University of Zagreb, Croatia. The original sample was stainless steel (BS 316S11, hardness: $160 \mathrm{VHN}$ ) and it was Tenifer bath nitride at $580^{\circ} \mathrm{C} / 4 \mathrm{~h}$ (T-SS, hardness: $300 \mathrm{VHN}$ ). Another SS sample was carbon steel (BS 060A62), vanadized at $950{ }^{\circ} \mathrm{C} / 4 \mathrm{~h}$ (V-CS, hardness: 3000 VHN).

The term Tenifer describes the nitrocarburizing process whereby nitrogen and carbon chemically released and introduced into the surface at a suitable high temperatures to allow the diffusion process to take place. The surface which has been treated, consists of two layers, called the compound layer and the lower diffusion layer. The former consists of carbonitrides and is between $12-16 \mu \mathrm{m}$ thick. The latter contains released nitrogen, which precipitates and forms needle-shaped nitrides only during slow cooling or annealing at over $300^{\circ} \mathrm{C}[14,15]$.

The vanadizing procedure is usually performed in a salt bath of special composition, which contains the carbideforming element vanadium, but might also contain some other elements, e.g. $\mathrm{Cr}, \mathrm{Nb}$. At elevated temperatures from 900 to $1000{ }^{\circ} \mathrm{C}$, a molten bath of borax containing vanadium, either in the form of metal or an oxide, results in atomic vanadium forming on the surface of the substrate, which is usually steel. Entering the austenite lattice, the vanadium diffuses from the surface into the substrate, up to the solubility limit, which depends on temperature and chemical composition of steel matrix. At this point, the reaction of vanadium and carbon results in the formation of vanadium carbides. Through the newly formed carbide layer the carbon diffuses outwards, where the process of forming carbides continues, thus increasing the thickness of the carbide layer [16-18].

\subsection{Micro-abrasion tests}

Micro-abrasion tests were performed with a commercially available apparatus, the TE-66, micro-abrasion tester (Phoenix tribology Ltd., UK). In this rig, as shown in Fig. 1, a rotating $25 \mathrm{~mm}$ diameter ball was in contact with a stationary sample, which was held between two-coaxial shafts, each carried by a support bearing. A variable speed DC motor was used to drive one shaft and a peristaltic pump, which is used to deliver the slurry from the storage vessel to the contact area. By selecting a constant number of revolutions, a test could be run for a fixed sliding distance. (For example, 150 revolutions correspond to linear sliding distance of $11.78 \mathrm{~m}$ ). The test sample was clamped onto a platform, which is fitted to the pivoted L-shaped arm. This arm was rotated around its pivot until the sample became in contact with the ball. The beam was in balance when the samples were in contact and the load was applied by adding dead weights to a cantilever arm. This configuration has the advantage of accurate control of both normal load (to an accuracy of $\pm 0.01 \mathrm{~N}$ ) and sliding speed.

The slurry consisted of $4 \mu \mathrm{m} \mathrm{SiC} \mathrm{particles,} \mathrm{in} \mathrm{distilled}$ water at concentration of $0.025 \mathrm{~g} \mathrm{~cm}^{-3}$, and was stored in a

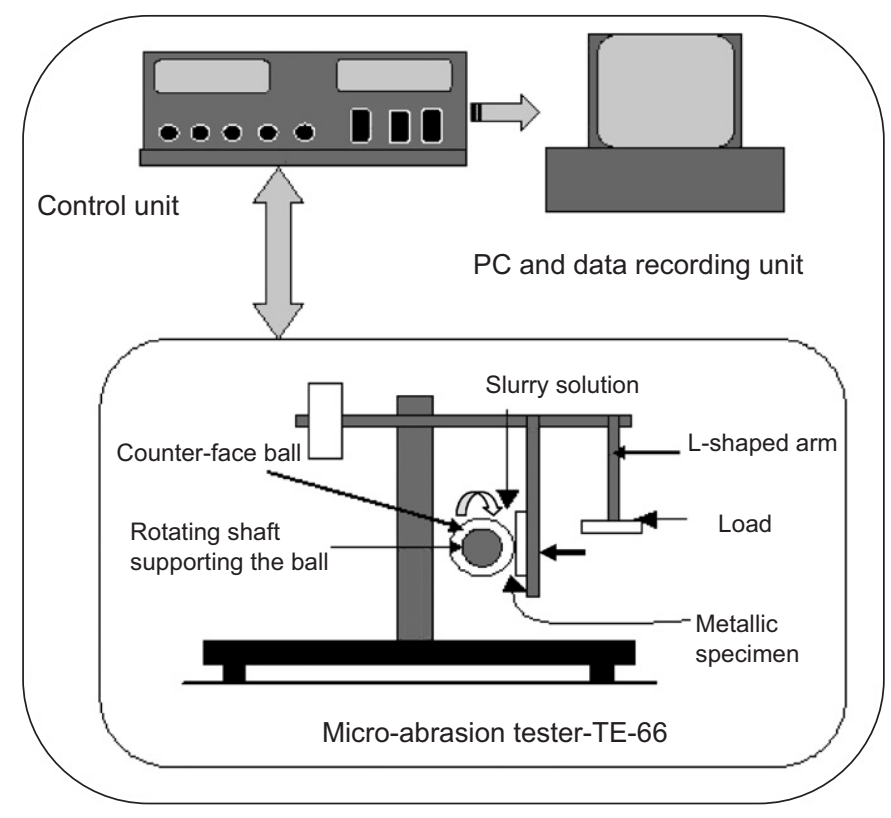

Fig. 1. Schematic diagram of microabrasion tester. 
Table 1

Micro-abrasion experimental details on thermo chemical treated steels

\begin{tabular}{|c|c|}
\hline $\begin{array}{l}\text { Sample } \\
\text { materials }\end{array}$ & $\begin{array}{l}\text { Stainless steel (SS), Tenifer bath nitrided stainless steel } \\
\text { (T-SS), vanadized carbon steel (V-CS), hardness values are } \\
160,300,3000 \mathrm{VHN} \text {, respectively at } 5 \mathrm{~kg} \text { load }\end{array}$ \\
\hline Ball material & $\begin{array}{l}\text { Stainless steel (SS- } 440 \mathrm{c} \text {, diameter- } 25 \mathrm{~mm} \text {, supplied by SKF } \\
\text { bearings, Hardness - } 750 \mathrm{VHN} \text { at } 5 \mathrm{~kg} \text { load) }\end{array}$ \\
\hline Speed & $100 \mathrm{rpm}$ or $0.13 \mathrm{~m} / \mathrm{s}$ \\
\hline Load & $1-5 \mathrm{~N}$ \\
\hline $\begin{array}{l}\text { Sliding } \\
\text { distance }\end{array}$ & $\begin{array}{l}150,375,750,1500,3000 \mathrm{rev} \text { or } 11.78,29.44,58.88,117.75 \\
235.50 \mathrm{~m}\end{array}$ \\
\hline Slurry & $\begin{array}{l}\text { Slurry composition: silicon carbide (SiC- } 4 \mu \mathrm{m} \text { diameter) } \\
\text { with distilled water (concentration of } 0.025 \mathrm{~g} \mathrm{~cm}^{-3} \text { ) }\end{array}$ \\
\hline
\end{tabular}

container that could be agitated on a laboratory magnetic stirrer. It was fed to a position just above the contact point and collected in a waste tray underneath, as explained earlier. The sample surfaces were ground and polished by conventional metallographic methods before testing (final grinding and polishing operations were carried out with 4000 grit size abrasive paper). The arm, which holds the sample, could be moved horizontally in order that several tests on a single sample specimen could be carried out. The sample was then removed from the apparatus and the diameters of the resulting abrasion scars were measured with a profile projector. Following the test, the worn samples were examined by optical microscopy and scanning electron microscopy. Other details about the samples and tested conditions are shown in Table 1.

The wear volume was calculated using the standard technique for measuring the wear scar of spherical geometry i.e., the geometry of the wear scar is assumed to reproduce the spherical geometry of the ball and the wear volume $(V)$ may then be calculated by the measurement of the crater diameter by a using profile projector [3],

$$
V=\frac{\pi b^{4}}{64 R} \text { for } b \ll R,
$$

where ' $b$ ' is the crater diameter and ' $R$ ' the radius of the ball.

\section{Results}

\subsection{Variation of wear volume as a function of applied load and sliding distance}

The micro-abrasion wear volume of the thermochemically treated steels, i.e., the untreated SS, T-SS and V-CS is shown in Fig. 2(a)-(e). The variation of wear volume at the lowest sliding distance i.e. $11.78 \mathrm{~m}$ is shown in Fig. 2(a). It is very clear that the T-SS shows high values of wear volume compared to the untreated SS. It is also interesting to note that the V-CS did not show any significant difference in micro-abrasion resistance compared with the un-treated material. For the T-SS, the variation of wear volume showed an increasing trend as a function of load at all sliding distances, with the exception of at $117.5 \mathrm{~m}$, Figs. 2(a), (b) and (d). For the V-CS and SS materials; there was no clear variation on the trends of wear with increasing load.

\subsection{Micrographs and SEM images of the abraded surfaces}

The SEM images of the SiC particles used in the test, Fig. 3(a), indicate its angular geometry. The SEM image of the worn surfaces, Fig. 3(b), on the SS at $5 \mathrm{~N}$ clearly shows a highly plastically deformed surface with the presence of grooves and ridges.

The un-abraded surfaces or the plane surface (before the test) of the T-SS is shown in Fig. 3(c). Fig. 3(d) shows some gouging features on the worn surfaces of T-SS at $5 \mathrm{~N}$ and a sliding distance of $235.5 \mathrm{~m}$. It also demonstrates that plastic deformation occurs on the surfaces during micro-abrasion as in the case of SS, Fig. 3(b). The un-abraded surface of the V-CS is shown in Fig. 3(e). The presence of grooves and ridges, Fig. 3(f), are also observed following the microabrasion process.

\section{Discussion}

\subsection{Variation of wear volume as a function of applied load and sliding distances}

The variation of wear volume as a function of applied load and sliding distance, Fig. 2(a)-(d), clearly shows that T-SS samples undergo severe micro-abrasion loss compared to the original SS and V-CS. The variation of wear volume with load over the three applied loads evaluated in this study is in accordance with the Archard relationship on wear rate [19]. However, there is some evidence of reduction in the wear volume at intermediate loads, which is addressed in more detail in Section 4.2.2.

In order to compare the performance of SS and V-CS, the studies were conducted by considering the penetration depth of wear scar, as shown in Fig. 4. The penetration depth, ' $d$ ' was calculated similar to the wear volume, based on the crater diameter ' $b$ ' and the radius of the ball, ' $R$ ' [3],

Penetration depth, $d=\frac{b^{2}}{8 R}$.

It is clear that, at shorter sliding distances, 11.78 and $58.88 \mathrm{~m}$, the V-CS shows low wear volume compare to others. At longer sliding distances, the wear resistance is similar to SS. This indicates that the beneficial effect on the wear rate of the carbide layer formed on the surface during heat treatment diminishes as the sliding distance is increased. It is clear that on reaching the penetration depth of around $10-14 \mu \mathrm{m}$, the micro-abrasion behaviour of V-CS is similar to that of the SS.

It should be noted that the selection of heat treatment processes has a significant effect on materials properties 


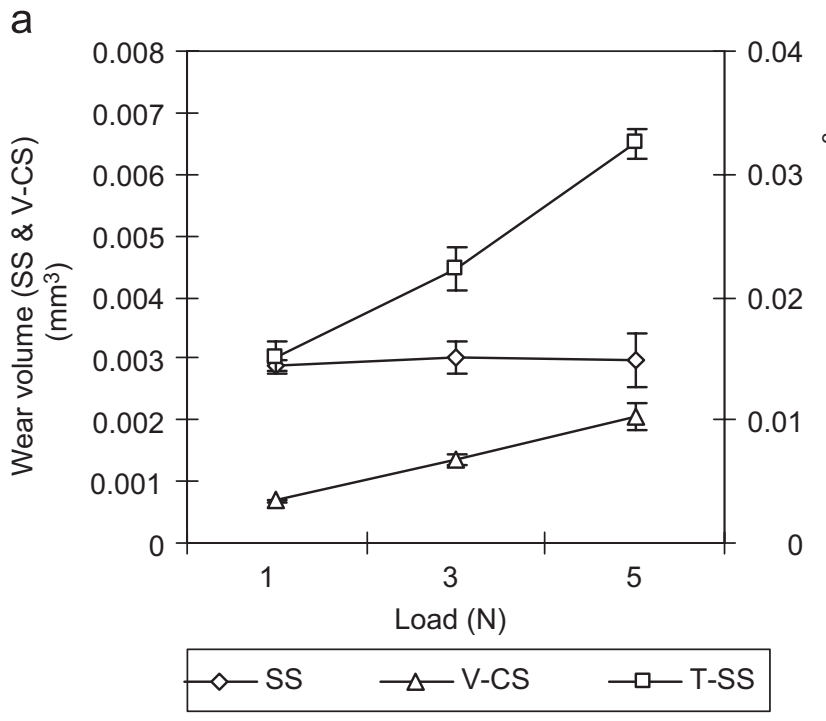

C

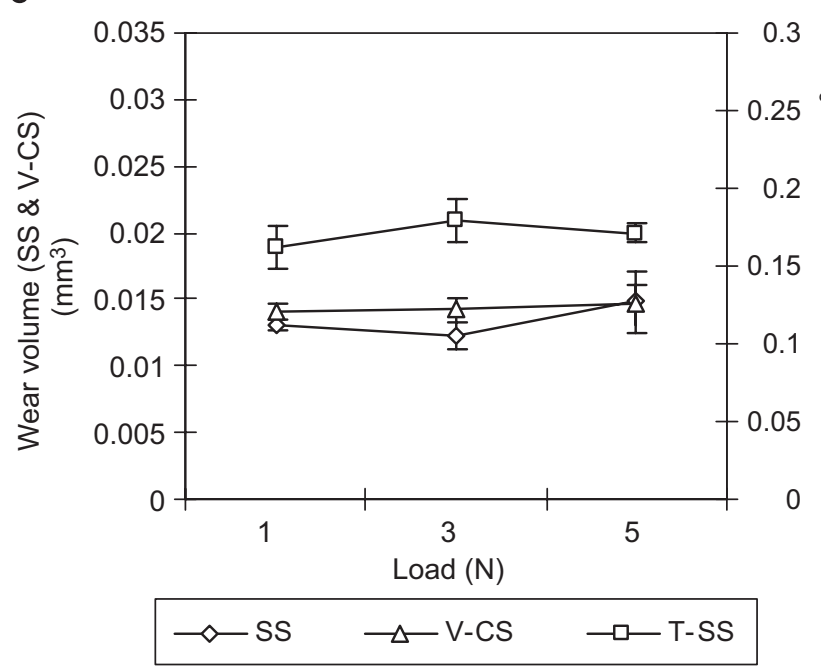

b

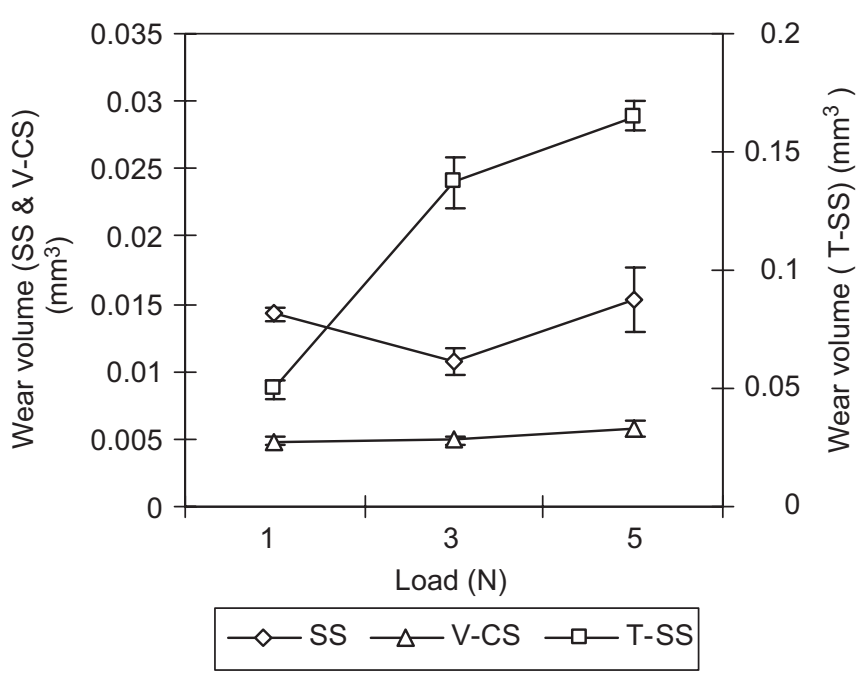

d

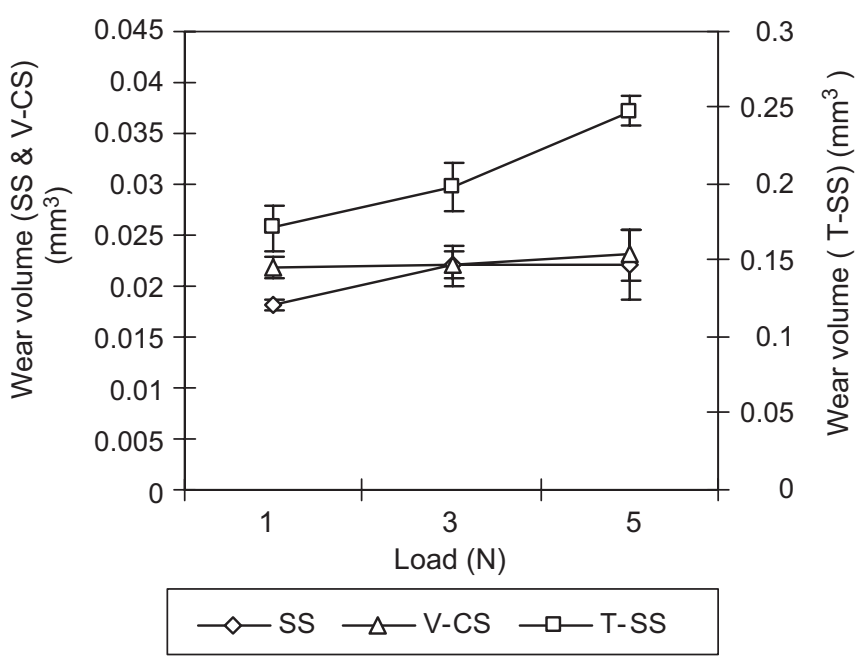

Fig. 2. (a)-(d) Variation of wear volume as a function of applied load at selected sliding distances for case hardened stainless steel: (a) 11.78; (b) 58.88; (c) 117.5 ; and (d) $235.5 \mathrm{~m}$.

such as hardness, toughness, strength, which in turn affects the wear resistance [20]. From the SEM images, Fig. 3(c) and (e), it is clear that the Tenifer bath nitride process, $\mathrm{T}$-SS, is a nitrocarburizing process as stated above, that produces a less compact structure compared to vanadized, V-CS, which is a surface deposition treatment at high temperature. Hence, the pre-treatment applied to the surface is also a reason for the poor micro-abrasion resistance of the T-SS.

In another study by Chiu et al. [21] on the wear behaviour of nitrocarburized tool steel, it was reported that such treatments increase the surface roughness, hardness and the friction coefficient. Further the duration of process did not have significant effect on wear resistance. It was also interesting to note that in other studies it was reported that the surface treatment may produce a solid lubricant film on the surface and resultant improvement of tribological properties, as indicated Zhang et al. [22]. Hence, the enhancement of abrasion resistance mainly depends on the type of thermochemical treatment applied to the steels. Other work shows that T-SS surface has good corrosion resistance [15] irrespective its poor microabrasion resistance.

In the current study, the hardness values of the T-SS and V-CS were 300 and $3000 \mathrm{VHN}$, respectively, as opposed to $750 \mathrm{VHN}$ for the stainless steel ball (SS-440c), Table 1. The anomalous behaviour, where the harder surface experiences higher wear rates during micro-abrasion, may be due to the harder steel pushing the particles into the counterface thus causing higher wear through a 2 body gouging process, than the softer steel (although it is not very clear with V-CS). Similar results have been found by Hogmark for ceramic coatings, where they experience abrasion rates significantly higher than the softer materials for this very reason [23]. Further, the hardness at the worn surface will generally be greater than the bulk hardness depends on the work hardening properties of the materials. In the case of case hardened steels with a very thin hardened layer, such 
a

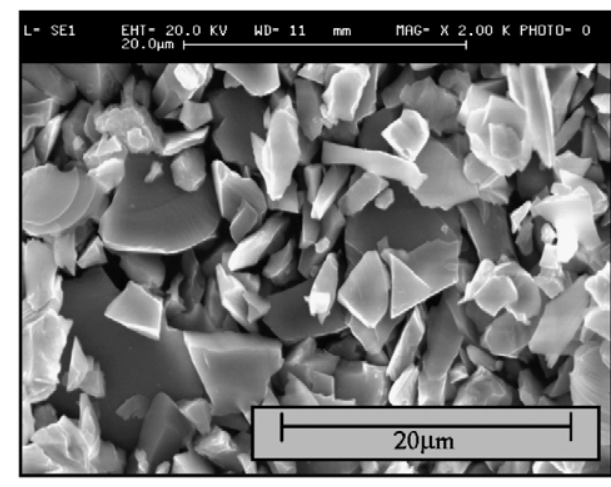

C

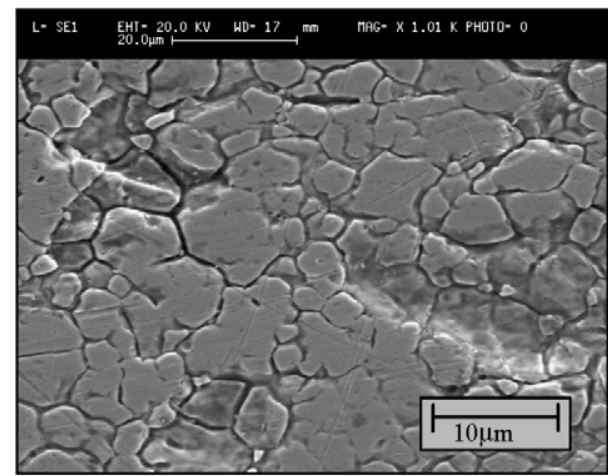

e

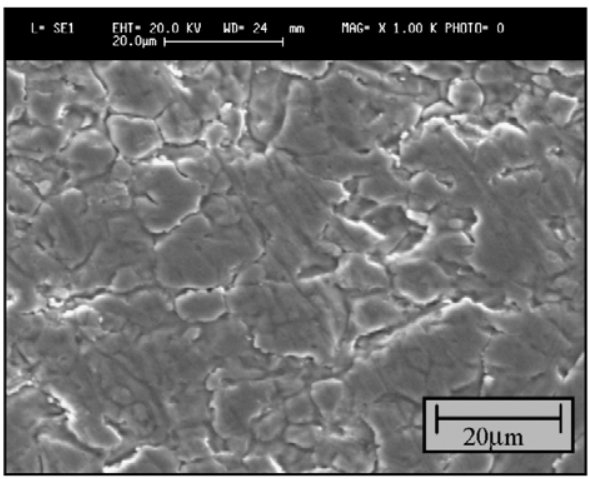

b

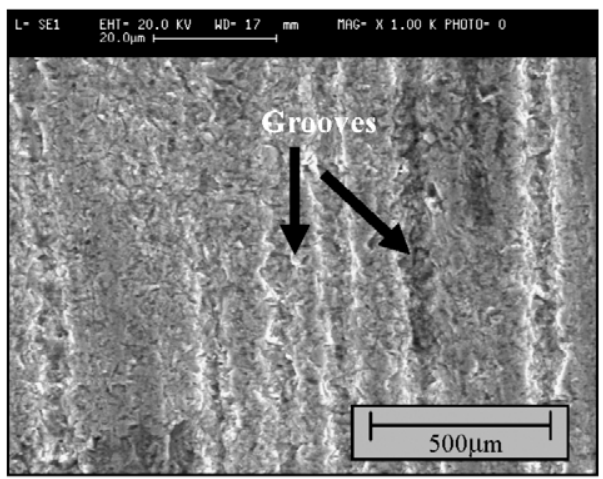

d

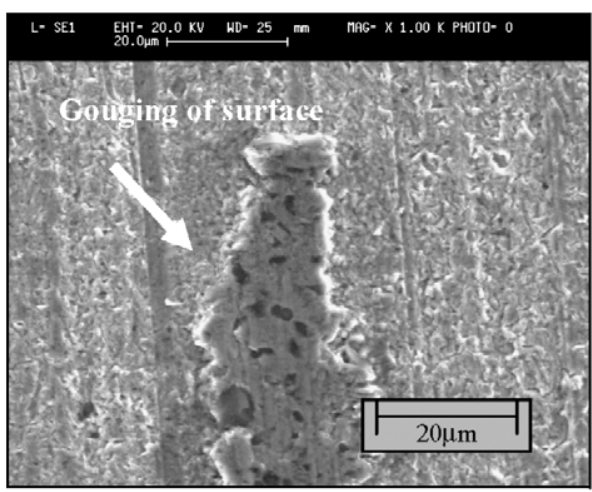

f

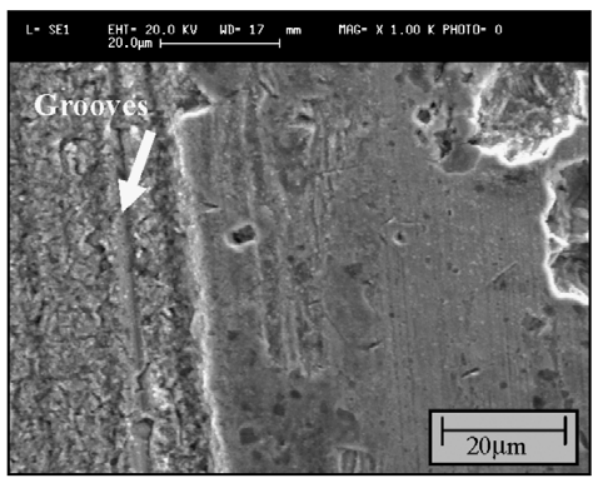

Fig. 3. (a)-(f) SEM images of the worn surfaces: (a) SiC-abrasive particles used in the tests; (b) $5 \mathrm{~N}, 235.5 \mathrm{~m}$, original SS; (c) plane surface, T-SS; (d) $5 \mathrm{~N}$, $235.5 \mathrm{~m}$, high magnification, T-SS; (e) Plane surface, V-CS; and (f) $5 \mathrm{~N}, 235.5 \mathrm{~m}$, V-CS.

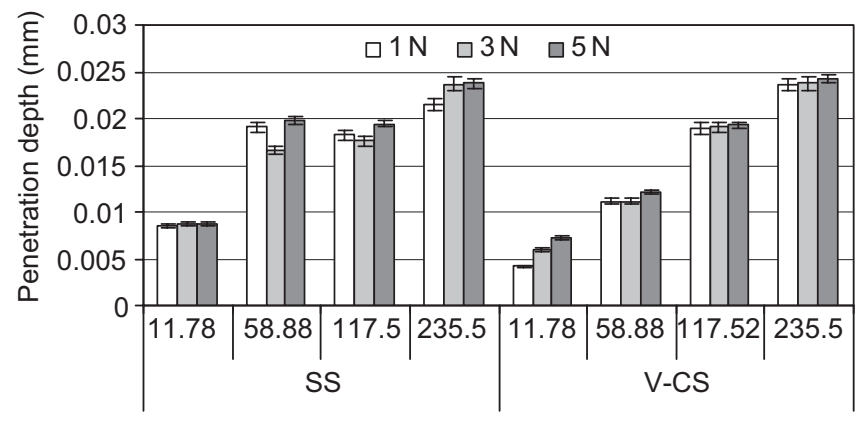

Sliding distance $(\mathrm{m})$

Fig. 4. Variation of penetration depth as a function of sliding distance and applied load for SS and V-CS. effects are very predominant in determining the hardness values and hence the wear resistance [24]. Consequently such effects of variation in hardness of contacting surfaces on micro-abrasion should not be neglected.

\subsection{Construction of the maps and possible mechanisms}

\subsubsection{Wastage maps}

In the earlier work [8-9], a schematic diagram of the possible occurrence of mechanisms (Fig. 5) involved in the micro-abrasion process was illustrated. Based on the similar approach, micro-abrasion mechanism and wastage maps for selected steels as a function of applied load and sliding distance was constructed as displayed in 
Figs. 6(a)-(c). The maps indicate schematically the severity (based on the wear volume) of the micro-abrasion process for a given conditions and mechanistic information on the wear loss mode. These tools are very helpful to understand the mechanisms of the wear process and how it affects the total wastage loss.

Three wastage regimes in the present map are defined as $[7,8]$
Low:
$V<0.01 \mathrm{~mm}^{3}$
Medium:
High

$$
\begin{aligned}
& 0.01 \mathrm{~mm}^{3} \leqslant V<0.05 \mathrm{~mm}^{3} \\
& V \geqslant 0.05 \mathrm{~mm}^{3}
\end{aligned}
$$

where ' $V$ ' denotes the wear volume. The selection of the criteria was based on earlier work of the authors [7-10] on the micro-abrasion behaviour of various classes of materials, ranging from pure metals to coatings and polymers.

It is clear from the maps that SS shows a low wastage zone at short sliding distances, and a similar pattern is observed in the case of V-CS. However, in the case of T-SS, the medium and high wastage zone predominate, indicating very poor performance in these micro-abrasion conditions. (It should be noted that if the severity of exposure conditions were reduced, i.e. if the load were reduced further, a transition to the low wastage regime could be anticipated; this was not addressed in the current work).

\subsubsection{Proposed mechanisms}

From Fig. 2(a)-(d), it is clear that there is some evidence of reduction in the wear volume at intermediate loads (see 2(b), SS, at $3 \mathrm{~N}$ and 2(c), SS at $3 \mathrm{~N}$ and T-SS at $5 \mathrm{~N}$ ), although these experiments were carried out for only three selected loads. This is in accordance with the mechanisms proposed by the authors [9] in previous work. Further to the mechanisms already proposed, such as grooving and rolling [12], it is suggested that the reduction in the wear volume at higher loads may be due to the presence of ridges and lubrication effects of the medium. In addition, there is also a likelihood of frictional heating and tribochemical effects at high loads. Hence, the mechanisms involved in the micro-abrasion process as shown in the schematic diagram Fig. 5 [8] can be summarized as below:

(i) 3-2 Body: gradual increment in the wear volume occurs as a function of applied load, which can be further subdivided as follows:

(a) Rolling: particles roll between the interacting surfaces.

(b) Grooving: particles adhere to the counter-body and leading to grooving.

(c) Mixed: this is a combination of the above two regimes.

(ii) 2-Body $r$ : reduction of the wear volume as a function of applied load, which includes the following scenarios:

(a) particles are entrained in the ridges and interacting surfaces may come in to contact with each other, resulting in to a 2-body wear process; (b) Lubrication effect of the water: this is a situation where particles disappear into the ridges and the asperities on top of the ridges are lubricated by the action of the carrier medium (in the current study, water). Hence, a reduction in wear rate might be expected, depending on the lubrication properties of the medium.

(c) Frictional heating and oxide films on the asperity contact: as is clear from the schematic diagram, in the absence of particles and medium, the contacting surfaces will commence to slide against each other under high contact stresses (which depends on the applied load). Such a situation may lead to frictional heating at the asperity contacts, resulting in oxide film formation due to tribochemical effects. This further complicates the sliding process as the oxide may have a beneficial or detrimental effect on the wear rate depending on its adhesion to the surface. However, additional analyses (the chemical analysis of worn surface for the oxide film formation or analysis of the solution after the test, for instance atomic absorption spectroscopy) are required to clarify such effects, which will be addressed in the next stage of the work.

The presence of 2-body $r$ mechanism in the current study is not very dominant as is indicated from Fig. 2(a)-(d); however, an attempt was made to analyse the predominance of such mechanisms. As is shown in Figs. 6(a)-(c), the circles and stars denote the 3-2 body and 2 body $r$ wear mechanisms.

It is clear that the 3-2 body region dominates in all the cases. The SS and T-SS show some slight evidence for the presence of the of the 2-body $r$ regions. It is interesting to note that in the case of the SS the 2-body $r$ regime is consistent with the low wastage zone that was observed at intermediate loads.

In fact, it should be noted that the occurrence of the micro-abrasion mechanisms, whether 3-2 body or grooving or 2-body $r$, is influenced by many other factors such as load, speed, sliding distance, properties of the contacting materials and abrasives, etc. Above all, the non-uniform distribution and concentration of slurry in the contacting surface is also a significant factor in micro-abrasion, because it may lead to slurry starvation at the contact zone $[12,13]$. Hence, the sequence of micro-abrasion mechanisms can be expected as shown in a schematic diagram, Fig. 7(a) and (b). It is expected that in the initial stage of the process, the 3-2 body abrasion mechanism dominates; further the micro-abrasion process progresses into the 2-body $r$ regime as explained earlier. It should also be noted that if the contact zone is considered under a broad perspective, these mechanisms can occur microscopically, i.e., locally, or macroscopically, i.e., globally in the contact zone, as illustrated in Fig. 7(b).

Moreover, the total wear volume loss caused by the micro-abrasion process, is dynamic, and depends on the 


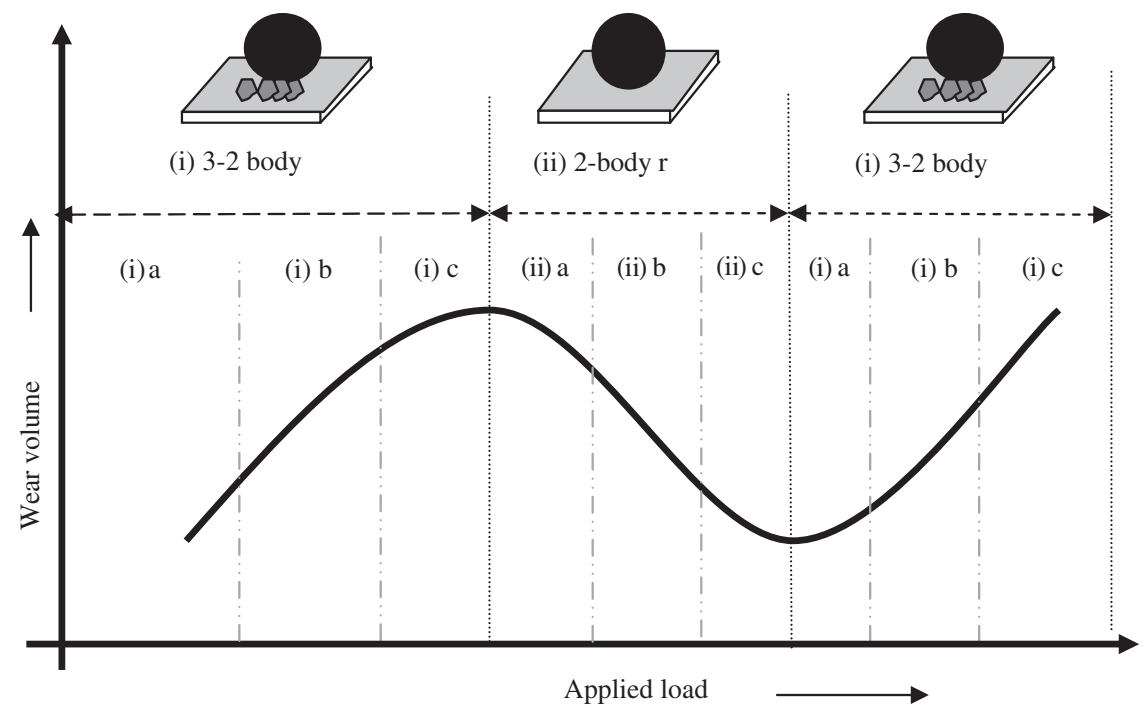

Fig. 5. Schematic diagram of transitions between the wear mechanisms as a function of increasing load [7-9].

a

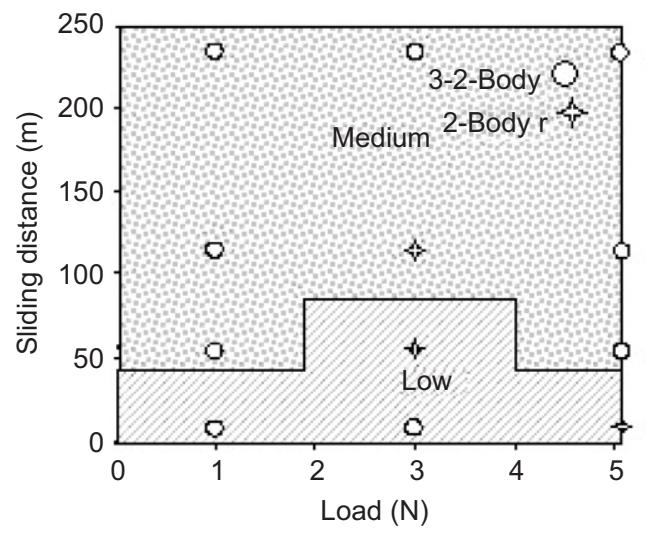

b

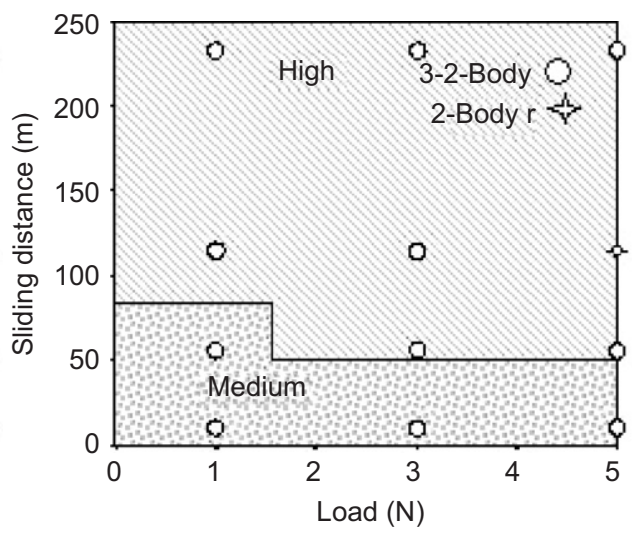

C

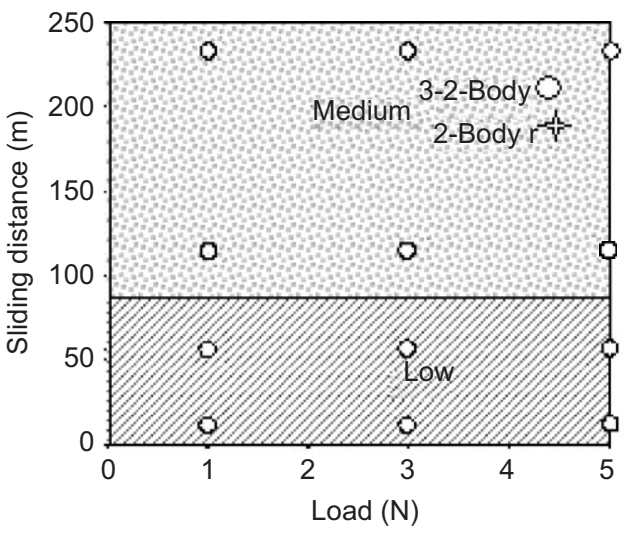

Fig. 6. (a)-(c) Micro-abrasion wastage maps for the tested samples (a) SS, (b) T-SS and (c) V-CS.

mechanisms dominating at that particular moment. As mentioned above they are in turn influenced by many other factors. The present study was focused only on the influence of the load and sliding distances. Undoubtedly, there is a need to consider other factors in future research, in order to have a clear understanding of the process.

\subsubsection{Material selection map}

A schematic material selection map, Fig. 8, has been developed by considering the low wastage zones of the individual wastage maps of the selected case hardened steels, Fig. 6(a)-(c). It is clear from the map that, the T-SS is completely eliminated from the map, and also, the SS 


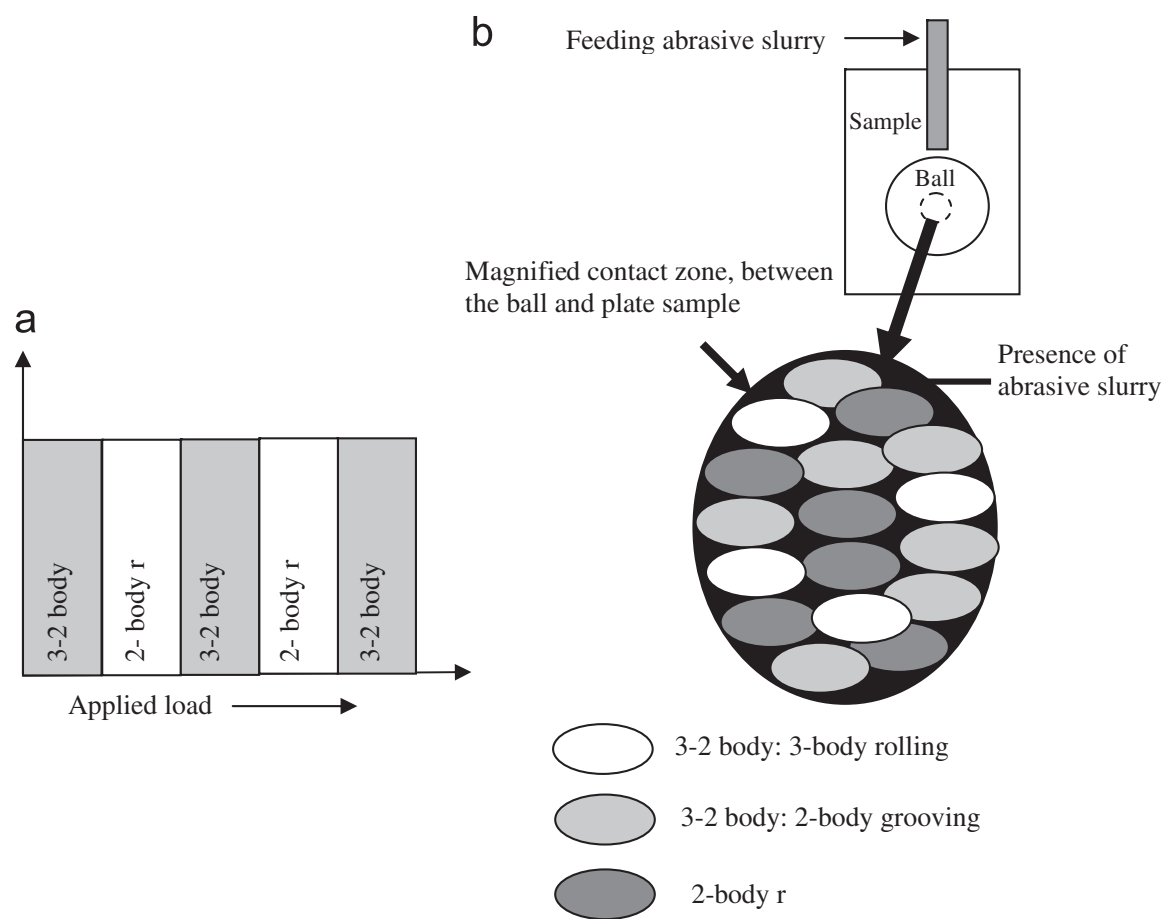

Fig. 7. (a) and (b) Schematic diagram of the presence of micro-abrasion mechanisms and process.

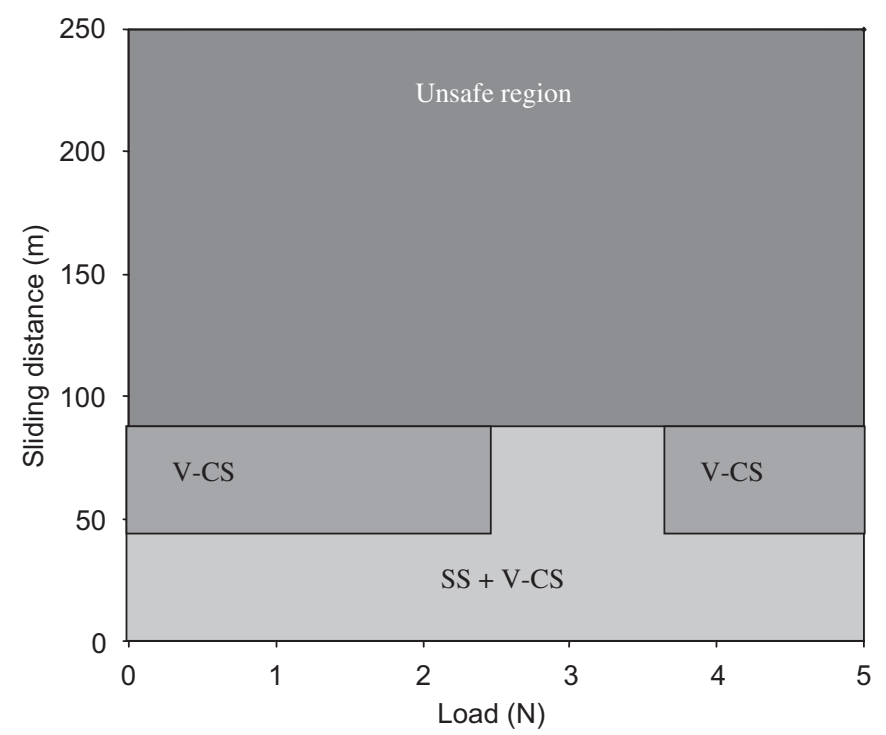

Fig. 8. Material selection map for the selected thermochemically treated steels.

and V-CS are located at shorter sliding distances irrespective of the increment in load. The vanadizing process has resulted in a slight improvement in the micro-abrasion performance of SS. As stated above, the selection of heat treatment process and other process parameters are significant prior to choosing a wear resistant material. Moreover, the structure of the formed hardened layer and its properties such as hardness and work hardening are also among the other influential parameters to be considered $[20,21]$.
It is worth in mentioning that, these maps, i.e., Figs. 6(a)-(c) and 8, are bound to the limitation of the experimental work and need to be extended by considering intermediate experimental conditions and other parameters (which may include sliding speed, type of solution and slurry and temperature, etc.), which affect the microabrasion process. However, in the current work the maps, which have been used are initial attempts in following the well established research work in employing mapping approaches in materials science [25-27].

\section{Conclusions}

The micro-abrasion resistance of two thermochemically treated case hardened stainless steels were analysed as a function of applied load and sliding distances, namely, T-SS and V-CS and their results were compared with the SS.

The possible mechanisms of micro-abrasion process have been discussed and based on the results in the study, microabrasion mechanisms and wastage maps were developed for the three steels.

Finally, a materials selection map was also developed in order to have a general understanding of the performance of the surface treated stainless steels in the micro-abrasion conditions evaluated in this study.

\section{Acknowledgement}

The authors would like to acknowledge, Material Science Department of Faculty of Mechanical Engineering 
and Naval Architecture, University of Zagreb, Croatia for providing the necessary samples.

\section{References}

[1] Rabinowicz E. Friction and wear of materials. New York: Wiley; 1965. p. 121-22.

[2] Eyre TS. Wear characteristics of metals. Tribol Int 1976;9(5):203-12.

[3] Rutherford KL, Hutchings IM. Theory and application of a microscale abrasive wear test. J Testing Evaluat 1997;25(2):250-60.

[4] Shipway PH, Hodge CJB. Micro-abrasion of glasses - critical role of ridge formation. Wear 2000;237:90-7.

[5] Sinnett-Jones PE, Wharton JA, Wood RJK. Micro-abrasion-corrosion of a CoCrMo alloy in simulated artificial hip joint environment. Wear 2005;259:898-909.

[6] Gant AJ, Gee MG. Abrasion of tungsten carbide hard metals using hard counterfaces. Int J Refract Met Hard Mater 2006;24(1-2): 189-98.

[7] Stack MM, Mathew MT, Jawan H. On the construction of microabrasion maps for a steels/polymer couple in corrosive environment. Tribol Int 2005;38(9):848-56.

[8] Stack MM, Mathew MT. Mapping the micro-abrasion resistance of WC/Co based coatings in aqueous conditions. Surf Coat Technol 2004;183(2-3):337-46.

[9] Stack MM, Mathew MT. Micro-abrasion transitions of metallic materials. Wear 2003;255:14-22.

[10] Stack MM, Mathew MT. Transitions in micro-abrasion mechanisms for HVOF coated steels. J Eng Tribol 2005;219(1):49-57.

[11] Adachi K, Hutchings IM. Sensitivity of wear rates in the micro-scale abrasion test to test conditions and material hardness. Wear 2005;258:318-21.

[12] Kusano Y, Hutchings IM. Sources of variability in the free-ball micro-scale abrasion test. Wear 2005;258:313-7.

[13] Shipway PH. A mechanical model for particle motion in the microscale abrasion wear test. Wear 2004;257:984-91.
[14] Cajner F, Landek D, Stupnisek Lisac E. Improvement of properties of steels applying salt bath nitrocarburizing with post-oxidation. Materiali in Tehnologije (1580-2949) 2003;37(6):333-9.

[15] Otmacic H, Tadic K, Stupnisek-Lisac E. Electrochemical investigation of thermally modified steel surfaces. In: Proceedings of the international workshop on "advanced technique for energy sources investigation and testing," 4-9 2004. Sofia, Bulgaria.

[16] Matijevic B, Stupnisek M. The diffusion growth of carbide layers on steel surfaces. Mater Technol 2000;34(6):237-40.

[17] Wang GB. Wear mechanisms in vanadium carbide coated steels. Wear 1997;212:25-32.

[18] Matijevic B, Stupnisek M. Diffusion forming of carbide layers to reduce abrasive wear. In: Proceedings of the 1st international conference on material \& tribology 2002, Dublin, Dublin Institute of Technology, 2002. CD.

[19] Archard JF. Contact and rubbing of flat surfaces. J Appl Phys 1953;24:981.

[20] Hemanth J. Wear characteristics of austempered chilled ductile iron. Mater Des 2000;21:139-48.

[21] Chiu LH, Wu CH, Chang $\mathrm{H}$. Wear behaviour of nitrocarburized JIS SKD61 tool steel. Wear 2002;253:778-86.

[22] Zhang N, Da-Ming Z, Jia-Jun L, Xiao-Dong F, Ming-Xi G. Wear mechanism of ion-sulphuriztion layer on steel under dry conditions. Wear 2001;247:1-8.

[23] Axen N, Jacobson S, Hogmark S. Influence of hardness of the counter-body in three-body abrasive wear-an overlooked harness effect. Tribol Int 1994;27(4):233.

[24] Hutchings IM. Tribology - friction and wear of engineering materials. Butterworth Heinemann; 1992.

[25] Ashby MF, Brechet YJM, Cebon D, Salvo L. Selection strategies for materials and processes. Mater Des 2004;25:51-67.

[26] Stack MM. Mapping tribo-corrosion process in dry and in aqueous conditions: some new directions for millennium. Tribol Int 2002;35:681-9.

[27] Brechet Y, Bessetti D, Landru D, Salvo L. Challenges in materials and process selection. Progr Mater Sci 2001;46:407-28. 